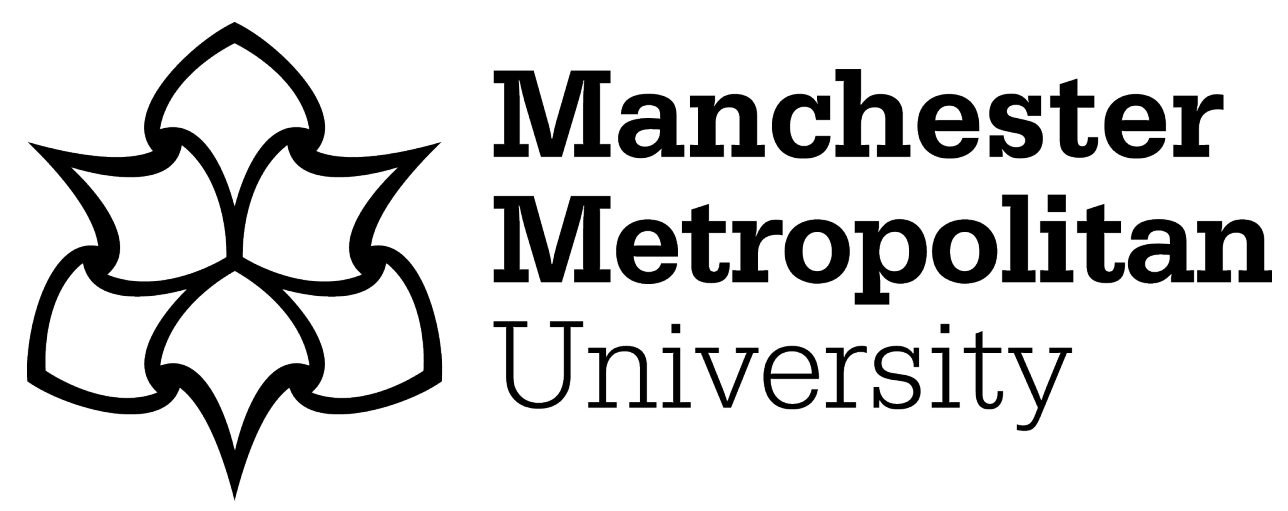

Tetlow, LA, Lynch, S and Whitehead, KA (2017) The effect of surface properties on bacterial retention: a study utilising stainless steel and TiN/25.65at.\%Ag substrata. Food and bioproducts processing, 102. pp. 332339. ISSN 0960-3085

Downloaded from: https://e-space.mmu.ac.uk/617874/

Version: Accepted Version

Publisher: Elsevier

DOI: https://doi.org/10.1016/j.fbp.2017.01.011

Usage rights: Creative Commons: Attribution-Noncommercial-No Derivative Works 4.0

Please cite the published version 


\section{Highlights}

- The TiN/25.65at.\%Ag coating was antimicrobial against bacteria

- The physicochemistry of the bacteria and surfaces influenced bacterial retention

- Multifractal analysis (MFA) did not affect the density of the bacteria

- MFA demonstrated that surface properties affected bacterial spread and clustering

- The surface properties influenced specific species : substratum interactions 


\title{
The effect of surface properties on bacterial retention: a study utilising stainless steel
}

\section{and TiN/25.65at.\%Ag substrata}

Louise A. Tetlow ${ }^{1}$, Stephen Lynch $^{2}$ and Kathryn A. Whitehead ${ }^{1 *}$

${ }^{1}$ School of Healthcare Science, Manchester Metropolitan University, Manchester M1 5GD, UK.

${ }^{2}$ School of Computing, Mathematics \& Digital Technology, Manchester Metropolitan University, Manchester M1 5GD UK.

Corresponding Author: K. A. Whitehead, School Healthcare Science, Manchester Metropolitan University, UK. Tel: +441612471157 E-mail: K.A.Whitehead@mmu.ac.uk

\begin{abstract}
The requirement for antimicrobial surfaces to control microorganisms for use in the food industries is increasing. A TiN/25.65at.\%Ag coating and a stainless steel (304 2R) surface were characterised for roughness parameters, chemistry and physicochemistry (PC). Microbiological analysis was performed to determine the antimicrobial efficacy and retention of Staphylococcus aureus and Escherichia coli on the surfaces. Zone of inhibition assays were successful against only E. coli on the TiN/25.65at.\%Ag coating. A bacterial respiratory assay demonstrated that the TiN/25.65at.\%Ag coating was antimicrobial against both bacteria. Retention assays demonstrated that the physicochemistry of the bacteria and surfaces influenced bacterial retention. Multifractal analysis of the retained bacteria demonstrated that the surface properties affected the spread and clustering, but not the density of the bacteria. This work suggests that surface properties influenced specific species : surface interactions and therefore surfaces need to be tailored to specific requirements depending on the environment and microorganisms to be targeted. This work may aid in the production of coatings or surfaces that may provide more hygienic conditions.
\end{abstract}


Keywords: Bacterial retention; surface hygiene; physicochemistry; antimicrobial; TiNAg; stainless steel.

\section{$1 \quad$ Introduction}

Biofouling is a critical problem that is currently being faced by many industries, including the food professions, and poses vast economic costs worldwide (Whitehead and Verran, 2009). Possible problems associated with surface fouling and subsequent biofilm formation include potential risks to food quality, product spoilage, biodeterioration and blockages of mechanical components, and risks towards the health of the consumer (Whitehead and Verran, 2015). Outbreaks of food poisoning pose a particular risk to vulnerable members of society, particularly the old, young or immunocompromised. In 2011, the Centres for Disease Control and Prevention (CDC) reported that foodborne disease caused a projected 48 million illnesses, 128,000 hospitalisations and 3000 deaths annually in the United States between 1996 and 2010 (Nyachuba, 2010; Schlisselberg and Yaron, 2013; Srey et al., 2013). Open work surfaces within the food industry are considered to be a potential source of microbial and organic contamination with a high risk of biotransfer between contaminated and clean products (Whitehead et al., 2015). Contamination of food produce can occur at any stage of the process (Srey et al., 2013). The attachment and adhesion of microbes to solid surfaces is an important step in the process associated with foodborne infections (Palmer et al., 2007) and in the formation of biofilms (Hori and Matsumoto, 2010). The correlation between the hydrophobicity of bacteria and surfaces has been described in some works as a primary driving force in the adhesion of microbes to surfaces (Zeraik and Nitschke, 2012). Combined with the interactions with other surface parameters such as the Lewis acid-base and van der Waals forces, hydrophobicity can result in preferential bacterial attachment, adhesion and retention to surfaces (Faille et al., 2002; Whitehead et al., 2015). Although some claims have been made that bacteria which are generally negatively charged will preferentially adhere to negatively 
charged surfaces (Zeraik and Nitschke, 2012), there is conflicting data in this area due to assays being carried out in a number of different ways and under different environmental conditions (Chae et al., 2006; Whitehead et al., 2015).

Surface topography and roughness is an important parameter to consider when investigating microbial retention and the hygienic status of surfaces. Previous investigations into the correlation between surface roughness and bacterial retention have proposed that there is a relationship between greater surface roughness and increases in the numbers of retained bacteria (Jullien et al., 2003; Whitehead et al., 2011). However, other works have found no relationship (Hilbert et al., 2003; Whitehead et al., 2005; Milledge, 2010) and suggestions have been offered that the current descriptive factors for roughness parameters are lacking or inappropriate (Zhao et al., 2008; Wickens et al., 2014). Many studies that examine the effect of surface properties on microbial retention use percentage coverage as a measure of retained bacteria on a surface (Al-Radha et al., 2012). However, mathematical packages such as MATLAB ${ }^{\circledR}$ allows for the use of multifractal analysis to give further information on the dispersion, distribution or clustering of the bacteria across the surfaces (Breki et al., 2016).

The aim of this research was to determine the effect of surface properties on the retention of two potentially pathogenic bacteria, and to determine the antimicrobial efficacy of a hardwearing TiN - silver containing coating when compared to conventionally used stainless steel. TiN was chosen as the carrier metal for silver due to its established use in many high wear, abrasive environments for example its use in drill bits components (Kelly et al., 2009).

\section{$2 \quad$ Materials and Methods}

\subsection{Production and analysis of surfaces and coatings}

304 stainless steel (Aalco, UK) was cut into $20 \mathrm{~mm}$ x $20 \mathrm{~mm}$ coupons. The side to be coated was prepared by the manufacturer with a $2 \mathrm{R}$ finish $\left(\mathrm{S}_{a} 25.3 \mathrm{~nm} \pm 0.6 \mathrm{~nm}\right)$. Prior to coating, the coupons were cleaned with isopropanol and methanol (Sigma, UK) and wiped with a fibre free 
cloth (Buehler, USA), before being placed inside the vacuum chamber to be sputter coated according to Wickens et al., (2014) using set parameters; Magnetron chamber evacuation 2.0 x $10^{-6} \mathrm{~Pa}$; Argon gas $99.99 \%$ purity flow rate $19.00 \mathrm{sccm}$; Operating pressure $0.24 \mathrm{~Pa}$; Titanium magnetron pulsed DC mode $1500 \mathrm{~W}: 20 \mathrm{kHz}$ pulse frequency (90\% duty); Silver magnetron, pulsed DC mode $100 \mathrm{~W}: 20 \mathrm{kHz}$ pulse frequency (90\% duty); Nitrogen gas, 503 $-504 \mathrm{~nm} ; 60 \%$ of the full metal signal.

\subsection{Surface characterisation}

Optical surface profiling (Zemetrics, USA) was performed on the surfaces to measure the topography and roughness which was quantified through calculation of the $\mathrm{S}_{a}$ value (arithmetic area average value over the complete 3D surface) (Zemaps software [version 1.14.38]). The average values of peaks and valleys from the line profiles was also taken $(n=10)$. Contact angle measurements for the surfaces were determined at room temperature using the sessile drop technique (Whitehead and Verran, 2009) and the physicochemical parameters were calculated through the surface tension parameters of the polar and apolar liquids as described by Van Oss (1993).

\subsection{Microbiology-Culture preparation}

The microorganisms Staphylococcus aureus NCTC 10788 and Escherichia coli NCTC 10418 were used for microbiological assays. Stock cultures were stored in the freezer at $-80{ }^{\circ} \mathrm{C}$. Cultures were thawed as required and inoculated onto brain heart infusion (BHI) agar (Oxoid, UK) and incubated for $24 \mathrm{~h}$ at $37^{\circ} \mathrm{C}$. Stock cultures were re-frozen following use. To maintain cell physiology inoculated plates were replaced every 4 weeks and stored in the fridge at $4{ }^{\circ} \mathrm{C}$. S. aureus and E. coli colonies were inoculated into sterile BHI broth (Oxoid, UK) (10 mL) and incubated overnight at $37^{\circ} \mathrm{C}$ in an orbital incubator at 150 repetitions per min (RPM) for $18 \mathrm{~h}$. Cultures were removed from incubation and the cells washed once by centrifuging at $3600 \mathrm{rpm}$ for $12 \mathrm{~min}$. The supernatant was removed and the cells were re-suspended in sterile purified 
water to an optical density of $1.0 \pm 0.05$ at $540 \mathrm{~nm}$ using a spectrophotometer (Jenway 6305, UK). Cells were enumerated from the determination of colony forming units $/ \mathrm{mL}(\mathrm{CFU} / \mathrm{mL})$ using serial dilutions These counts equated to $5.9 \times 10^{8}( \pm 2.9) \mathrm{CFU} / \mathrm{mL}$ for $S$. aureus and 6.6 x $10^{8}( \pm 2.2) \mathrm{CFU} / \mathrm{mL}$ for E. coli.

\subsection{Microbial adhesion to hydrocarbons (MATH) assay}

The microbial adhesion to hydrocarbons (MATH) assay was followed as previously described by Whitehead et al., (2005). Cultures were prepared by inoculating $100 \mathrm{~mL}$ of brain heart infusion broth (Oxoid, UK) and incubated for $18 \mathrm{~h}$ at $37{ }^{\circ} \mathrm{C}$. Cells were harvested by centrifugation (3000 RPM) and washed in PUM buffer [pH 7.1] (22.2 g of potassium phosphate trihydrate (BDH, UK), $7.26 \mathrm{~g}$ of monobasic potassium phosphate (BDH, UK), $1.8 \mathrm{~g}$ of urea (Sigma, UK), and $0.2 \mathrm{~g}$ of magnesium sulfate heptahydrate (BDH, UK) / L) three times before being re-suspended to an optical density of 1.0 at $400 \mathrm{~nm}$. A volume of $1.2 \mathrm{~mL}$ of the cell suspension was added to round bottomed glass test tubes $(15 \mathrm{~mm})$ before $200 \mu \mathrm{L}$ of either chloroform (Sigma Aldrich, USA), ethyl acetate (Sigma Aldrich, USA), hexadecane (Sigma Aldrich, USA) or decane (Sigma Aldrich, USA) was added. Each sample was mixed using a vortex mixer for 2 min and was then left to stand at room temperature for 15 min to allow separation of the two phases. The lower aqueous phase of the mixture was removed and the optical density recorded. The calculation used to determine affinity to hydrocarbons was taken from Rosenberg et al., (1980);

$$
\text { Adherence }=\left(1-\frac{\mathrm{A}}{A_{0}}\right)
$$

Where $A_{0}$ was the optical density measured at $400 \mathrm{~nm}$ prior to mixing and $A$ was the absorbance after mixing.

\subsection{Zones of inhibition}

Coupons were cleaned in ethanol (Sigma, UK), wiped with a fibre free cloth (Buehler, USA) and dried in a class 2 cabinet before being adhered to the bottom of sterile Petri dishes with 
double sided sticky tape (Guilbert Niceday, UK). Cell solutions were prepared as above with $5 \times 10^{5}$ and $\mathrm{CFU} / \mathrm{mL}$, and $1.25 \mathrm{~mL}$ was added to $23.75 \mathrm{~mL}$ of molten brain heart agar. The solution of cells and agar was agitated gently to disperse the cells evenly before being gently poured over the coupons and cooled to set. The samples were incubated at $37^{\circ} \mathrm{C}$ for $24 \mathrm{~h}$ before the zones of inhibition were measured using digital Vernier callipers (Mitutoyo CD- 6" CP, Japan).

\subsection{Cell viability (Nitro tetrazolium violet) Assay}

The NTV assay methodology was followed as previously described by Wickens et al., (2012) and adapted to use Nitro tetrazolium violet as a safer option to Nitro tetrazolium blue. Cultures were prepared as above and diluted to give a solution of $10^{5} \mathrm{CFU} / \mathrm{mL}$. The diluted cell suspension $(10 \mu \mathrm{L})$ was pipetted and spread onto the individual coupons and dried in a class II airflow cabinet for $1 \mathrm{~h}$. Once dry, $25 \mathrm{~mL}$ of molten $\left(50^{\circ} \mathrm{C}\right)$ brain heart infusion agar (Oxoid, Basingstoke, UK) was poured gently over the coupons and allowed to cool before incubating at $37{ }^{\circ} \mathrm{C}$ for $24 \mathrm{~h}$. Following incubation, two $\mathrm{mL}$ of $0.01 \%$ filter sterilised Nitro Tetrazolium Violet (Sigma-Aldrich, UK) was flooded onto the top of the agar and incubated at room temperature for $6 \mathrm{~h}$. Viable colonies were visible as dark violet colonies which allowed counting for quantitative data and photographs were taken from qualitative data.

\subsection{Retention assays}

Overnight cell cultures were washed and re-suspended to OD 1.0 at $540 \mathrm{~nm}$, corresponding to concentrations of $S$. aureus $(6.8 \pm 2.2) \times 10^{8}$ and $E$. coli $(7.1 \pm 1.9) \times 10^{8} \mathrm{CFU} / \mathrm{mL}$. Two replicate substrata were place horizontally into a Petri dish to which $30 \mathrm{~mL}$ of the standardised cell suspension was added and incubated for $1 \mathrm{~h}$ without shaking. Following incubation, the coupons were removed with sterile forceps and each washed gently with $5 \mathrm{~mL}$ of sterile filtered water from a bottle at a $45^{\circ}$ angle with a $3 \mathrm{~mm}$ nozzle. The samples plus retained bacteria were then air-dried in a class II airflow cabinet for $1 \mathrm{~h}$ (Whitehead and Verran, 2007). To allow 
visualisation with an epifluorescence microscope, the samples were stained for 2 min with 0.03 $\%$ acridine orange (Sigma, USA) diluted in $2 \%$ glacial acetic acid (BDH, UK), rinsed in sterile distilled water and allowed to dry once more in a class II airflow cabinet in the dark for $30 \mathrm{~min}$. The retained cells upon the surface were visualised using epifluorescence microscopy (Nikon Eclipse E600 epifluorescence microscope, Tokyo, Japan) at $502-526 \mathrm{~nm}$ wavelength. Cell-F software was used to visualise, capture and analyse the images. These images were used in the MATLAB ${ }^{\circledR}$, Image Processing Toolbox ${ }^{\circledR}$ for the multifractal analysis $(n=20)$.

\subsection{Multifractal analysis}

This analysis is a further development of the method previously described in Wickens et al., (2014). The properties of typical multifractal spectra, the multifractal datasets were constructed from a set of motifs using MATLAB ${ }^{\circledR}$. Matrix (datasets) of size $512 \times 512$ were computed by overlaying the given motifs one on top of another. This resulted in $4 \times 4$ matrix being formed. Following a second iteration an $8 \times 8$ matrix was formed until completion. The MathWorks Image Processing Toolbox ${ }^{\circledR}$, was used to convert the datasets into grey scale images. On this scale, a value of zero would be black and a value of one would be white. The numerical $f(\alpha)$ spectra were computed for, $-10 \leq \mathrm{q} \leq 10$. Boxes of size $\varepsilon=4,8,16,32,64,128$ and 256 , were used to cover the datasets. Using the data cursor in MATLAB ${ }^{\circ}$, the two points $\alpha_{\max }$ and $\alpha_{\mathrm{min}}$ were estimated and used to give a measure of asymmetry $\left(\Delta \alpha_{A S}\right)$ was given by

$\Delta \alpha_{A S}=\frac{\alpha_{0}-\alpha_{\min }}{\alpha_{\max }-\alpha_{0}}$

Where the $\mathrm{f}(\alpha)$ curve is symmetric if $\Delta \alpha_{A S}=1$, left-skewed if $\Delta \alpha_{A S}>1$ (gaps) and rightskewed if $\Delta \alpha_{A S}<1$ (clustering of bright pixels, or cells in this case). The height of the alpha curves demonstrated the heterogeneity of the cells spread across the surfaces, whilst the width of the curves demonstrated cell density. Curve skewness demonstrates the number of cell clusters on the surfaces.

\subsection{Statistical analysis}


Statistical analysis was carried out using one way ANOVA and Student $t$-tests. The results were reported as \pm standard error. The differences observed between the substrates were considered significant at $p<0.05$.

\section{$3 \quad$ Results}

\subsection{Surface characterisation}

White light profilometry was performed to provide quantitative analysis of the surfaces roughness. The scans performed allowed visualisation of the surfaces demonstrating linear striations and pits on both the stainless steel and TiN/25.65at.\%Ag (Figure 1). Line profiles demonstrated that the stainless steel substrates possessed wider valleys $(3.21 \mathrm{~nm} \pm 0.5 \mathrm{~nm})$ than the TiN/25.65at.\%Ag $(1.9 \mathrm{~nm} \pm 0.5 \mathrm{~nm})$ but the width of the smaller valleys were similar between the two surfaces $(0.56 \mathrm{~nm} \pm 0.04 \mathrm{~nm}$ and $0.62 \mathrm{~nm} \pm 0.03 \mathrm{~nm}$ respectively) (Figure 1 and Table 1). The average peak height for both the stainless steel and TiN/25.65at.\%Ag substrates were similar in size, the peak height of the TiN/25.65at.\%Ag being slightly greater $(23.2 \mathrm{~nm} \pm 4.8 \mathrm{~nm}$ and $29.3 \mathrm{~nm} \pm 5.9 \mathrm{~nm}$ respectively). The peak to valley ratio of the stainless steel had a lower value than the TiN/25.65at.\%Ag surfaces demonstrating that the TiN/25.65at.\%Ag surfaces had larger peak sizes than the stainless steel. However, quantification of the $S_{a}$ value (the arithmetic average roughness across the surface) (Table 1) demonstrated no significant difference between the test replicates (stainless steel $=25.27 \mathrm{~nm}$, $\mathrm{TiN} / 25.65 \mathrm{at} . \% \mathrm{Ag}=29.06 \mathrm{~nm})(p=0.575)$.

The physicochemical results of the surfaces (Figure 2a) demonstrated the TiN/25.65at.\%Ag surface was hydrophilic $\left(6.54 \mathrm{~mJ} / \mathrm{m}^{2}\right)$ and the stainless steel was marginally hydrophobic ($\left.6.28 \mathrm{~mJ} / \mathrm{m}^{2}\right)$. The surface free energy $\left(\gamma_{s}\right)$ values demonstrated that TiN/25.65at.\%Ag had significantly greater SFE values $\left(57.73 \mathrm{~mJ} / \mathrm{m}^{2}\right)$ than the stainless steel controls $\left(40.73 \mathrm{~mJ} / \mathrm{m}^{2}\right)$. Lifshitz van der Waals $\left(\gamma^{L W}\right)$ values of the TiN/25.65at.\%Ag surface $\left(43.02 \mathrm{~mJ} / \mathrm{m}^{2}\right)$ was significantly greater than the stainless steel surface $\left(36.23 \mathrm{~mJ} / \mathrm{m}^{2}\right)$. The acid-base component 
of the surfaces $\left(\gamma^{A B}\right)$ showed that the TiN/25.65at.\%Ag coated samples had a higher $\gamma^{A B}$ value $\left(14.71 \mathrm{~mJ} / \mathrm{m}^{2}\right)$ than the stainless steel surface $\left(4.49 \mathrm{~mJ} / \mathrm{m}^{2}\right)$. The $\gamma^{+}$values of the surfaces demonstrated the electron accepting potential of the surfaces. The addition of the TiN/25.65at.\%Ag coating increased the electron accepting potential of the surfaces $(35.37$ $\left.\mathrm{mJ} / \mathrm{m}^{2}\right)$ in comparison to the stainless steel substrate $\left(0.21 \mathrm{~mJ} / \mathrm{m}^{2}\right)$, and the $\gamma^{-}$(electron donating) properties values of the surfaces were lower on the TiN/25.65at.\%Ag surface $\left(1.53 \mathrm{~mJ} / \mathrm{m}^{2}\right)$ compared to the stainless steel surface $\left(24.07 \mathrm{~mJ} / \mathrm{m}^{2}\right)$.

\subsection{Microbiology}

Microbial adhesion to hydrocarbons (MATH) assays were performed on S. aureus and E. coli to quantify their physicochemistries (Figure 2b). Results showed that S. aureus demonstrated a stronger affinity to chloroform $(99.87 \%)$ and hexadecane $(81 \%)$ than it did to ethyl acetate $(41.73 \%)$ and decane $(81.53 \%)$. E. coli also demonstrated a greater affinity to chloroform and hexadecane than to ethyl acetate and decane $(64.57 \%, 4.40 \%, 29.37 \%$ and $0 \%$ respectively). This demonstrated that both microbes were electron donors. E. coli demonstrated a very low affinity to hexadecane, which when paired with its greater affinity towards chloroform and ethyl acetate showed that it was more hydrophilic in nature than $S$. aureus. S. aureus demonstrated a high affinity towards the apolar solvents (hexadecane and decane) demonstrating that it was the more hydrophobic of the two bacteria tested.

Zones of inhibition testing the surfaces demonstrated that as expected, the stainless steel surfaces produced no zones of bacterial clearance against E. coli or $S$. aureus, hence no antimicrobial efficacy (Table 2). TiN/25.65at.\%Ag also produced no measurable zone of inhibition against $S$. aureus, however it did produce a statistically significant zone of inhibition against $E$. coli suggesting that $E$. coli was more susceptible towards the antimicrobial action of silver. Nitro tetrazolium violet assays demonstrated that both microorganisms were susceptible 
to the antimicrobial action of the TiN/25.65at.\%Ag surfaces (Table 2), and that between the microorganisms this result was statistically significant $(p=0.002)$.

Retention assays were performed demonstrated that there was no significant difference in the number of cells retained on the different test substrates (Figure 3 and 4). However, a trend was observed whereby $S$. aureus demonstrated greater numbers of retained bacteria upon the stainless steel surfaces than the TiN/25.65at.\%Ag surfaces whilst $E$. coli exhibited the opposite effect.

$f$ alpha curves were used to extrapolate the data with MATLAB (Figure 5). It was demonstrated that E. coli was more heterogeneously spread on TiN/25.65at.\%Ag than on stainless steel (Figure 6a). The opposite effect was found with $S$. aureus which was most heterogeneously spread on stainless steel. There was no difference observed in the cell density between the substrata for the same bacteria although the density of $S$. aureus was lower than for E. coli (Figure 6b). The most dispersed cells with the least clusters were E. coli on the TiN/25.65at.\%Ag surface whereas E. coli on stainless steel or S. aureus on stainless steel or TiN/25.65at.\%Ag demonstrated similar numbers of groupings (Figure 6c).

\section{Discussion}

Stainless steel was coated with TiN/25.65at.\%Ag to determine the effect of the surface properties (chemistry, topography and physicochemistry) on the antimicrobial activity and bacterial retention to the surface. Stainless steel and TiN/25.65at.\%Ag surfaces were tested for potential antimicrobial action. Antimicrobial testing upon the surfaces demonstrated that the stainless steel surfaces did not produce any zones of inhibition as predicted. The TiN/25.65at.\%Ag surfaces did produce a clear zone of inhibition against E. coli but not against S. aureus. This could be due to the thinner peptidoglycan wall of Gram-negative bacteria ( $E$. coli) in comparison to Gram-positive bacteria (S. aureus). Work by Ahern et al., (1995) used ion beam assisted silver, silver oxide and silver chloride surfaces and although they 
demonstrated that the surfaces inhibited both bacterial growth and bacterial adherence, they also found that the silver surfaces did not produce zones of inhibition in agar diffusion tests as did Mclean et al., (1993) using $\mathrm{Ag} / \mathrm{Cu}$ surfaces. It was proposed that rather than an antimicrobial effect caused by ion leaching that the Ag operates by promoting a catalytic interaction with oxygen which promoted bactericidal activity (Heining, 1993). The NTV respiratory assay demonstrated that as expected, the stainless steel produced no inhibition of the bacteria, whilst when in contact with the surface, the TiN/25.65at.\%Ag surfaces did produce a significant reduction in the number of colonies against both bacterial species, demonstrating that the TiN/25.65at.\%Ag surfaces did have antimicrobial mechanisms against both $E$. coli and $S$. aureus but that the method of testing and thus antimicrobial delivery was important. Others have hypothesized upon the mechanisms of the antimicrobial action of silver, suggesting that the silver ions caused structural changes in the bacterial cell walls disrupting the permeability and respiration of the cell, affected the interactions of thiol groups in proteins and enzymes, or created interruptions in DNA replication (Feng et al., 2000; Morones et al., 2005; Rai et al., 2009; Skovager et al., 2013). Again, the differences in bacterial cell wall morphology between Gram positive and Gram negative bacteria could explain why the mechanism for antimicrobial action and results produced by the TiN/25.65at.\%Ag surfaces were different between the bacterial species used in this study, since $S$. aureus is Gram-positive whilst E. coli is Gram-negative. Similarly, a study by Kelly et al., (2009) examined the effect of differing Ag concentrations in TiN/25.65at.\%Ag surfaces upon Pseudomonas aeruginosa and $S$. aureus and demonstrated that the surfaces that contained higher concentrations of silver had a greater bactericidal effect upon Gram-negative bacteria. Cell surface area and shape could also be factors contributing towards antimicrobial efficacy as a cell with a larger surface area, such as the rod shaped E. coli, could potentially be in contact with more silver ions from the TiN/25.65at.\%Ag surfaces than the cocci shaped S. aureus (Guzman et al.,, 2012). 
Analysis of the surfaces by white light profilometry demonstrated that there was no statistically significant difference in the surface roughness $\left(S_{a}\right)$ between the two surfaces. Line profile traces of the surfaces did reveal differences in the size and distribution of the peaks that made up the surface topography since the stainless steel surfaces possessed wider valleys than the TiN/25.65at.\%Ag substrates which could provide larger sites for retention and adhesion of bacteria (Flint et al., 2000; Hilbert et al., 2003; Hsu et al., 2013). However, in our work, surface features were not thought to influence bacteria retention. Differences in the surface chemistry, topography or roughness parameters can potentially change the physicochemical parameters of the surfaces for example, the electrostatic interactions at the cell: surface interface (Hsu et al.,, 2013) which therefore affects the retention of bacteria. Changes in the hydrophobicity of the surfaces are important when considering bacterial adhesion (Zeraik and Nitschke, 2012) since it may affect preferential binding to surfaces by different bacterial species (Whitehead and Verran, 2009). The TiN/25.65at.\%Ag was found to be more hydrophilic than the stainless steel surfaces. Sinde and Carballo (2000) reported that the presence of a hydrophobic substrata would favour bacterial adhesion, however, others have found the opposite (Chae et al., 2006) and have suggested that other factors must also be considered when looking at the physicochemistry of a surface and bacterial adhesion such as electrostatic surface charge, roughness and the properties of the bacterial cell (Whitehead and Verran, 2009; Zeraik and Nitschke, 2012; Skovager et al., 2013). Both the Lewis acid-base values and the Lifshitz van der Waals values are expressions representing the sum of either the attractive or repulsive forces acting upon the substrates, but from either a polar or apolar perspective respectively. The Lifshitz van der Waals, acid-base and electron accepting forces were found to be greater for the TiN/25.65at.\%Ag surface than the stainless steel wereas the electron donating forces were found to be greatest for the stainless steel surface. 
Assessment of the electron donating/accepting potential and hydrophobicity of the microorganisms has been suggested to be essential for assessing the hygienic status of the substrates (Bellon-Fontaine et al., 1996) and for predicting patterns of microbial attachment. The microbial adhesion to hydrocarbons assay enabled calculation of the physicochemistry for the bacterial species used in this study. This demonstrated that whilst both microbes were electron donors, $S$. aureus was hydrophobic and E. coli was more hydrophilic in nature. When analysing the bacterial retention data in conjunction with the physicochemistry of both the bacteria and substrates, trends emerged between the bacterial species. The hydrophobic $S$. aureus showed preference towards the more hydrophobic stainless steel, whilst the hydrophilic E. coli showed a preference for the hydrophilic TiN/25.65at.\%Ag. These results are comparable with research by Whitehead et al., (2015) in which it was found that E. coli was most affected by the physicochemistry of the surfaces. Works by others have documented that hydrophobicity is one of the primary driving forces involved in the adhesion of pathogens to surfaces (Sinde and Carballo, 2000; Faille et al., 2002; Zeraik and Nitschke, 2012). However, other have found no correlation in physicochemical parameters between the surfaces and bacteria (Chae et al., 2006; Whitehead and Verran, 2009; Whitehead et al., 2015). Whitehead et al., (2015) also found that $S$. aureus retention was also influenced by the topography of the surfaces, which was not demonstrated within this study.

Multifractal analysis of the bacteria on the different surfaces demonstrated a more in depth insight into the distribution of the bacteria on the surfaces. E. coli was spread more heterogeneously on TiN/25.65at.\%Ag whilst $S$. aureus was most heterogeneously distributed across the stainless steel. The TiN/25.65at.\%Ag surface also had an effect on E. coli which demonstrated the most dispersed cells with the least clusters which is consistent with a more hydrophilic surface. This is consistent with the hydrophilic surface demonstrating a 'wetting' effect. However, the substrata did not affect the cell density of either bacteria with respect to 
the surface properties. Surface hydrophobicity influenced the heterogeneity of the spread of the cells across the surfaces, but the pattern was opposite for the two bacteria. Thus it may be speculated that the density of the cells across the surfaces was not affected by the surfaces properties but by cell type. In agreement with this work, Wickens et al., (2014) demonstrated that S. aureus was more heterogeneously spread across surfaces than Staphylococcus epidermidis, and that the effects of the surface properties on bacterial retention were bacterial species specific. Thus, the use of multifractal analysis provides an effective tool in providing information of how surface properties affect microbial distribution across the surfaces.

\section{Conclusion}

A greater understanding of how surface properties affect microbial retention may help in the development and production of hygienic surfaces. The TiN/25.65at.\%Ag surface was demonstrated to be antimicrobial. The results of the study concluded that for the two species of bacteria used upon these surfaces, hydrophobicity was the main driving force for affected bacterial retention, and that within this instance, bacteria preferred surfaces with similar hydrophobicity characteristics to their own. These properties affected the spread and clumping of the cells. Cell density was affected by cell type. Thus, these surface properties affected the bacterial retention of the different species in very specific ways. This work suggests that the type of surface influences specific species : surface interactions and therefore surfaces need to be tailored to specific requirements depending on the environment and microorganisms to be targeted. Further research into the effects of topography upon these bacterial species would be required to enable conclusions to be drawn upon its effects on bacterial retention. The use of multidisciplinary research that incorporate systems such as multifractal analysis will allow researchers to further understand these complex interactions that may enable the development of surfaces to tackle individual fouling scenarios.

\section{Acknowledgements}


The authors would like to thank Manchester Metropolitan University for funding this work. We would also like to thank the surface engineering group for their support in making these surfaces.

\section{Conflict of interest}

None.

\section{References}

Al-Radha, A.S.D., Dymock, D., Younes, C., and O’Sullivan, D. (2012) Surface properties of titanium and zirconia dental implant materials and their effect on bacterial adhesion. $J$. Dent. 40: 146-153.

Breki, C.-M., Dimitrakopoulou-Strauss, A., Hassel, J., Theoharis, T., Sachpekidis, C., Pan, L., and Provata, A. (2015) Fractal and multifractal analysis of PET-CT images of metastatic melanoma before and after treatment with ipilimumab.

Chae, M.S., Schraft, H., Truelstrup Hansen, L., and Mackereth, R. (2006) Effects of physicochemical surface characteristics of Listeria monocytogenes strains on attachment to glass. Food Microbiol. 23: 250-259.

Faille, C., Jullien, C., Fontaine, F., Bellon-Fontaine, M.-N., Slomianny, C., and Benezech, T. (2002) Adhesion of Bacillus spores and Escherichia coli cells to inert surfaces: role of surface hydrophobicity. Can. J. Microbiol. 48: 728-738.

Feng, Q.L., Wu, J., Chen, G.Q., Cui, F.Z., Kim, T.N., and Kim, J.O. (2000) A mechanistic study of the antibacterial effect of silver ions on Escherichia coli and Staphylococcus aureus. J. Biomed. Mater. Res. 52: 662-668.

Flint, S.H., Brooks, J.D., and Bremer, P.J. (2000) Properties of the stainless steel substrate, influencing the adhesion of thermo-resistant streptococci. J. Food Eng. 43: 235-242.

Guzman, M., Dille, J., and Godet, S. (2012) Synthesis and antibacterial activity of silver nanoparticles against gram-positive and gram-negative bacteria. Nanomedicine 
Nanotechnology, Biol. Med. 8: 37-45.

Hilbert, L.R., Bagge-Ravn, D., Kold, J., and Gram, L. (2003) Influence of surface roughness of stainless steel on microbial adhesion and corrosion resistance. Int. Biodeterior. Biodegradation 52: 175-185.

Hori, K. and Matsumoto, S. (2010) Bacterial adhesion: From mechanism to control. Biochem. Eng. J. 48: 424-434.

Hsu, L.C., Fang, J., Borca-Tasciuc, D.A., Worobo, R.W., and Moraru, C.I. (2013) Effect of micro-and nanoscale topography on the adhesion of bacterial cells to solid surfaces. Appl. Environ. Microbiol. 79: 2703-2712.

Jullien, C., Bénézech, T., Carpentier, B., Lebret, V., and Faille, C. (2003) Identification of surface characteristics relevant to the hygienic status of stainless steel for the food industry. J. Food Eng. 56: 77-87.

Kelly, P.J., Li, H., Whitehead, K.A., Verran, J., Arnell, R.D., and Iordanova, I. (2009) A study of the antimicrobial and tribological properties of TiN/Ag nanocomposite coatings. Surf. Coatings Technol. 204: 1137-1140.

Milledge, J.J. (2010) The cleanability of stainless steel used as a food contact surface: an updated short review. Food Sci. Technol. J. 24: 27-28.

Morones, J.R., Elechiguerra, J.L., Camacho, A., Holt, K., Kouri, J.B., Ramirez, J.T., and Yacaman, M.J. (2005) The bactericidal effect of silver nanoparticles. Nanotechnology 16: $2346-2353$.

Nyachuba, D.G. (2010) Foodborne illness: is it on the rise? Nutr Rev 68: 257-269.

Van Oss, C.J. (1993) Acid—base interfacial interactions in aqueous media. Colloids Surfaces A Physicochem. Eng. Asp. 78: 1-49.

Palmer, J., Flint, S., and Brooks, J. (2007) Bacterial cell attachment, the beginning of a biofilm. J. Ind. Microbiol. Biotechnol. 34: 577-88. 
Rai, M., Yadav, A., and Gade, A. (2009) Silver nanoparticles as a new generation of antimicrobials. Biotechnol Adv 27: 76-83.

Rosenberg, M., Gutnick, D., and Rosenberg, E. (1980) Adherence of bacteria to hydrocarbons: A simple method for measuring cell-surface hydrophobicity. FEMS Microbiol. Lett. 9: 29-33.

Schlisselberg, D.B. and Yaron, S. (2013) The effects of stainless steel finish on Salmonella Typhimurium attachment, biofilm formation and sensitivity to chlorine. Food Microbiol. 35: $65-72$.

Sinde, E. and Carballo, J. (2000) Attachment of Salmonella spp. and Listeria monocytogenes to stainless steel, rubber and polytetrafluorethylene: the influence of free energy and the effect of commercial sanitizers. Food Microbiol. 17: 439-447.

Skovager, A., Whitehead, K., Wickens, D., Verran, J., Ingmer, H., and Arneborg, N. (2013) A comparative study of fine polished stainless steel, TiN and TiN/Ag surfaces: Adhesion and attachment strength of Listeria monocytogenes as well as anti-listerial effect. Colloids Surfaces B Biointerfaces 109: 190-196.

Srey, S., Jahid, I.K., and Ha, S.-D. (2013) Biofilm formation in food industries: A food safety concern. Food Control 31: 572-585.

Taylor, J.H. and Holah, J.T. (1996) A comparative evaluation with respect to the bacterial cleanability of a range of wall and floor surface materials used in the food industry. $J$. Appl. Bacteriol. 81: 262-266.

Verran, Packer, A., Kelly, P., and Whitehead, K.A. (2010) Titanium-coating of stainless steel as an aid to improved cleanability. Int J Food Microbiol 141, Suppl: S134-S139.

Whitehead, K.A., Benson, P.S., and Verran, J. (2015) Developing application and detection methods for Listeria monocytogenes and fish extract on open surfaces in order to optimize cleaning protocols. Food Bioprod. Process. 93: 224-233. 
Whitehead, K.A., Benson, P.S., and Verran, J. (2011) The detection of food soils on stainless steel using energy dispersive X-ray and Fourier transform infrared spectroscopy. Biofouling 27: 907-17.

Whitehead, K.A., Colligon, J., and Verran, J. (2005) Retention of microbial cells in substratum surface features of micrometer and sub-micrometer dimensions. Colloids Surfaces B Biointerfaces 41: 129-138.

Whitehead, K.A. and Verran, J. (2009) The Effect of Substratum Properties on the Survival of Attached Microorganisms on Inert Surfaces. In, Flemming,H.-C., Murthy,P.S., Venkatesan,R., and Cooksey,K. (eds), Marine and Industrial Biofouling. Springer Berlin Heidelberg, pp. 13-33.

Whitehead, K.A. and Verran, J. (2007) The effect of surface properties and application method on the retention of Pseudomonas aeruginosa on uncoated and titanium-coated stainless steel. Int. Biodeterior. Biodegradation 60: 74-80.

Wickens, D., Lynch, S., West, G., Kelly, P., Verran, J., and Whitehead, K.A. (2014) Quantifying the pattern of microbial cell dispersion, density and clustering on surfaces of differing chemistries and topographies using multifractal analysis. J Microbiol Methods 104: 101-108.

Wickens, D.J., West, G., Kelly, P.J., Verran, J., Lynch, S., and Whitehead, K.A. (2012) Antimicrobial activity of nanocomposite zirconium nitride/silver coatings to combat external bone fixation pin infections. Int. J. Artif. Organs 35: 817-25.

Zeraik, A.E. and Nitschke, M. (2012) Influence of growth media and temperature on bacterial adhesion to polystyrene surfaces. Brazilian Arch. Biol. Technol. 55: 569-576.

Zhao, Q., Liu, Y., Wang, C., Wang, S., Peng, N., and Jeynes, C. (2008) Reduction of bacterial adhesion on ion-implanted stainless steel surfaces. Med Eng Phys 30: 341-349. 

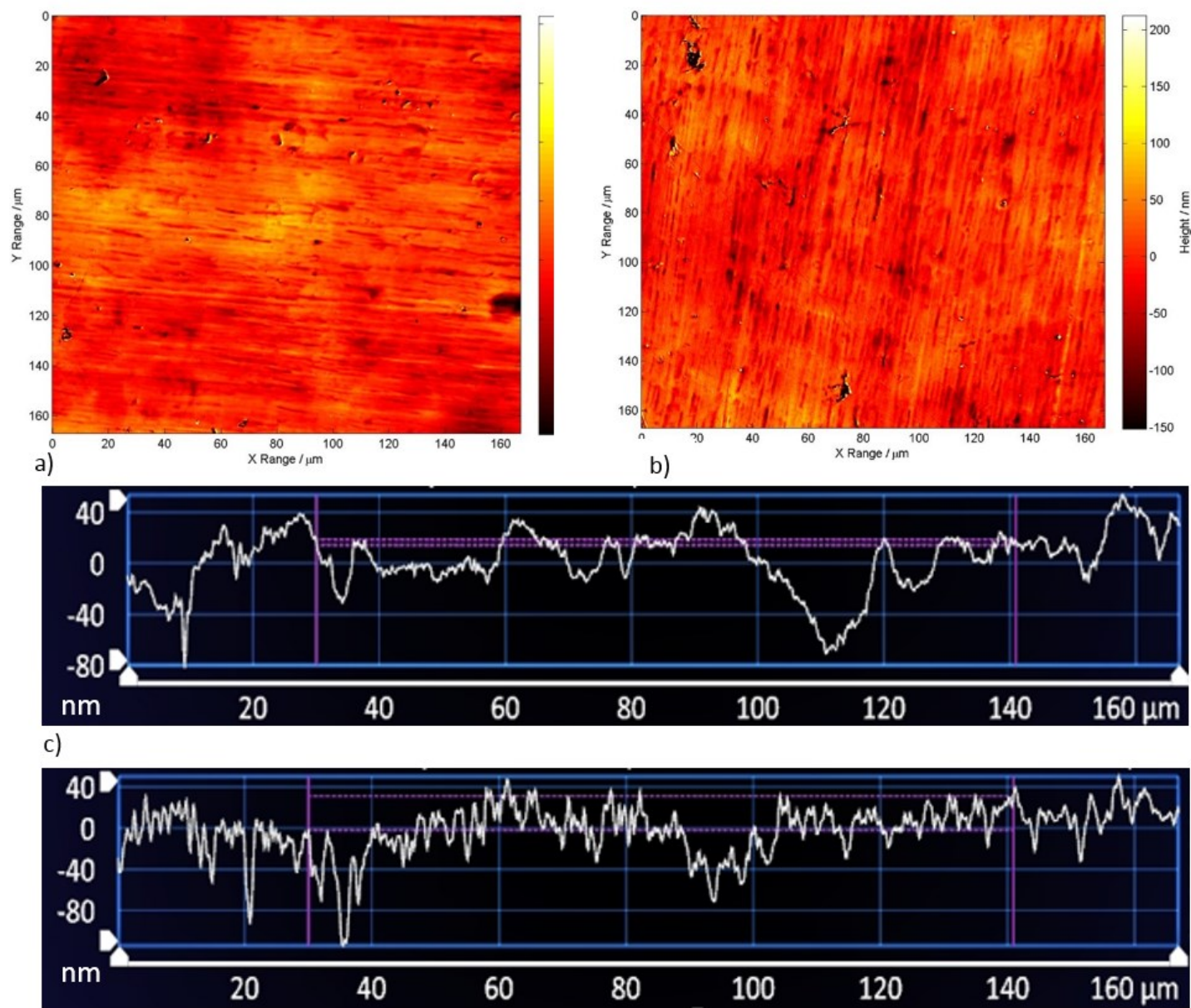

d)

Figure 1: White light profilometry pictures describing the surface topography of a) stainless steel and b) TiN/25.65 at. \% Ag coupons and line profiles from the WLP scans of c) Stainless steel and d) TiN/25.65 at. \% Ag surfaces displaying a cross-sectional profile of the width and depth of the peaks and valleys upon the surfaces. 


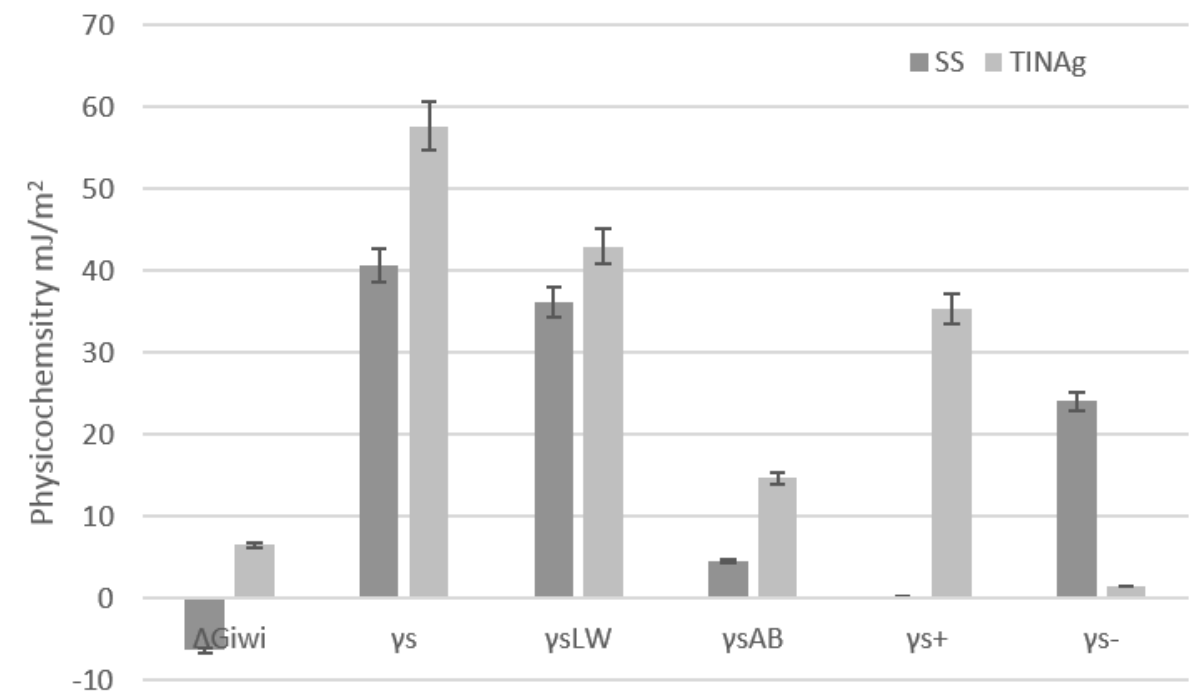

Fig. 2. Physicochemistry assays demonstrating a) the surface energy parameters for both the stainless steel and TiN/25.65 at. \% Ag surfaces, b) MATH assay showing the percentage affinity of the microorganisms toward the different solvents, demonstrating the physicochemistry of the different bacteria. 


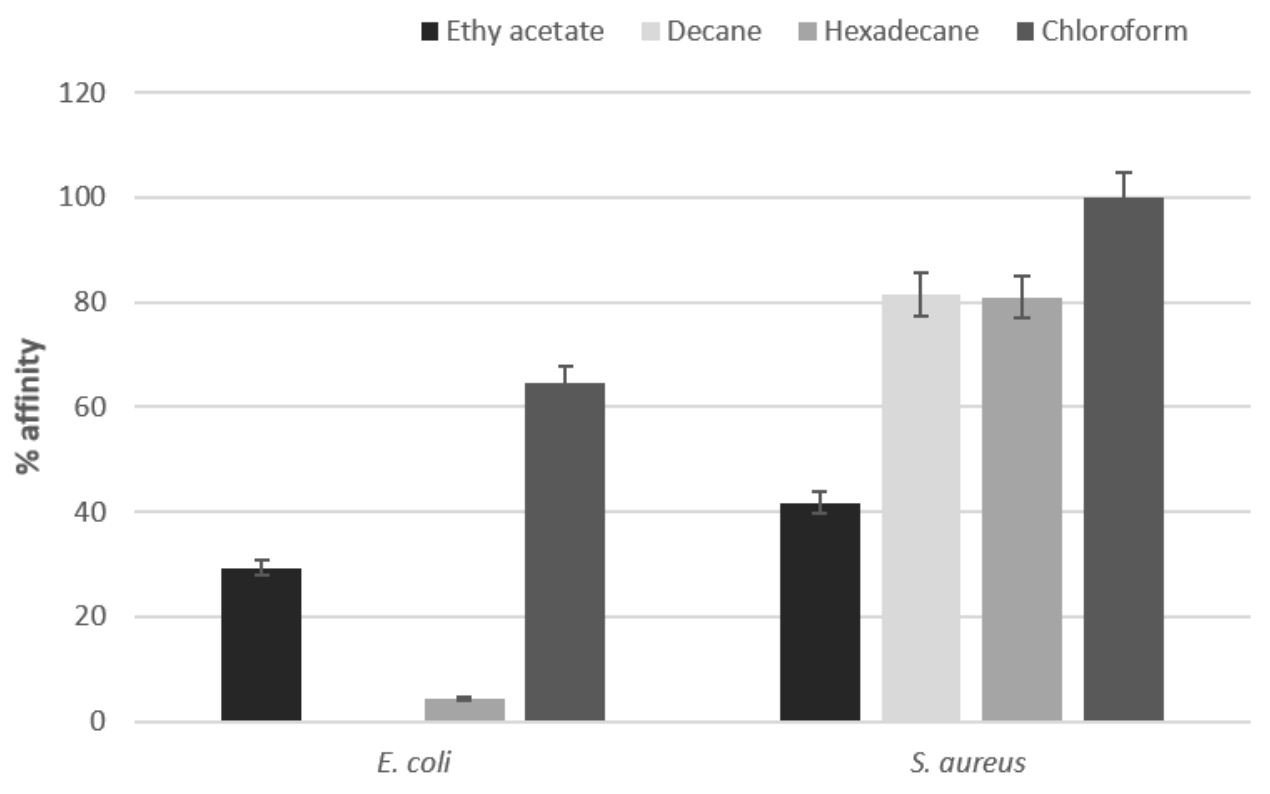

Fig. 3. Epifluorescence images depicting the retention a) E. coli on stainless steel, b) E. coli on TiN/25.65 at. \% Ag, c) S. aureus on stainless steel, d) S. aureus on TiN/25.65 at. \% Ag coupons demonstrating the different patterns of retained bacteria on each of the test substrates. 

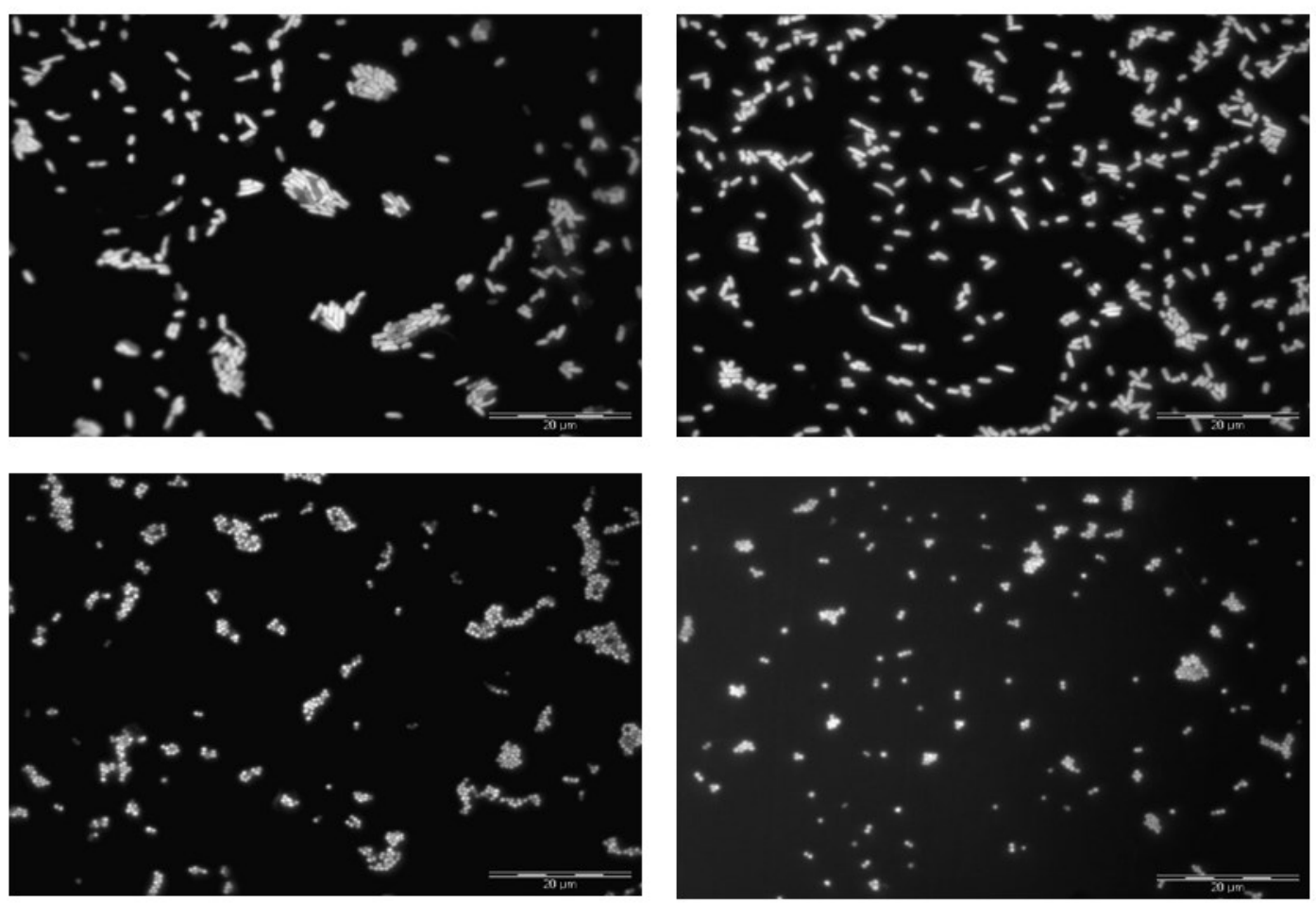

Fig. 4. Retention assays performed on stainless steel and TiN/25.65 at.\% Ag coupons using $S$. aureus and E. coli. S. aureus demonstrated greater numbers of retained bacteria upon the stainless steel surfaces than the TiN/25.65 at.\% Ag surfaces, E. coli exhibited greater preference for the TiN/25.65 at.\% Ag surfaces. 


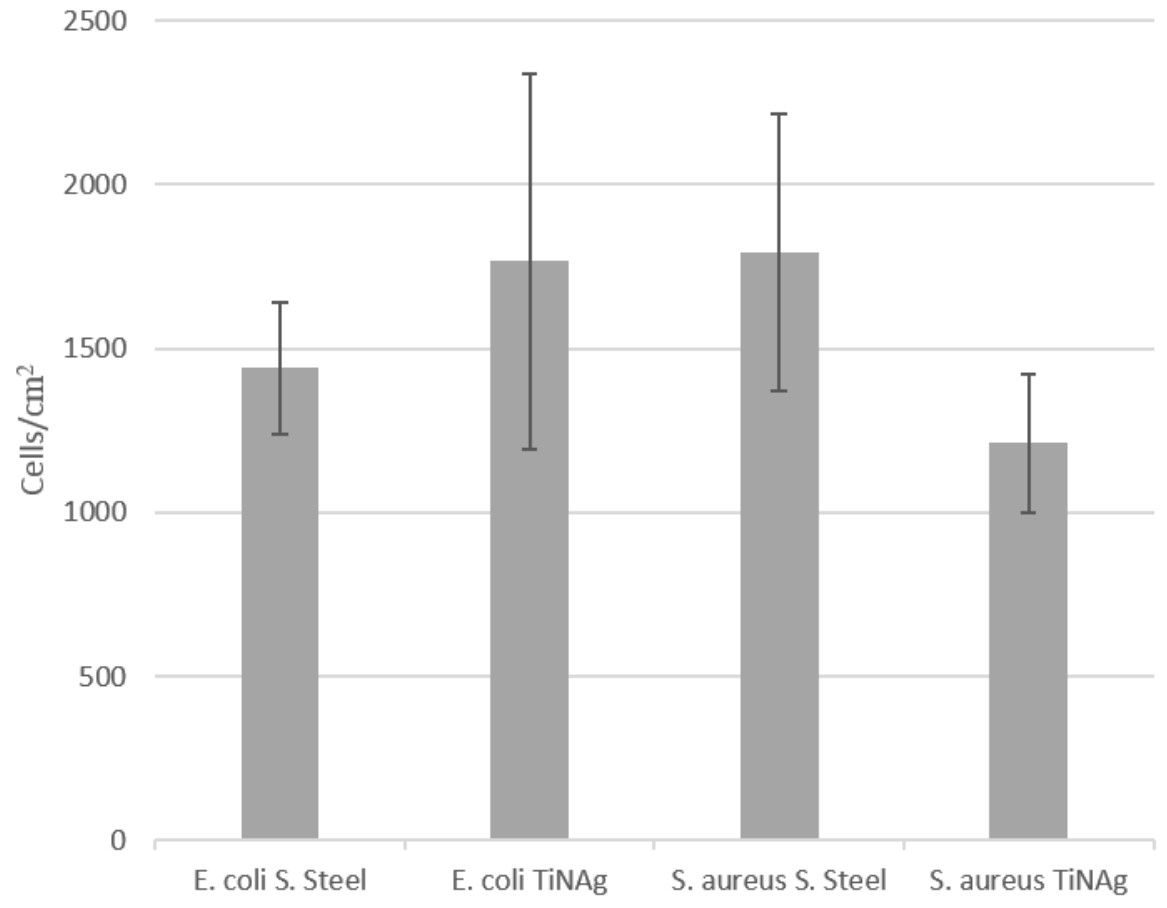

Fig. 5. Examples of $f$ alpha curves demonstrating how the data is extrapolated to describe the distribution and pattern of cells on the surfaces. E. coli is denoted by the red line and S. aureus is denoted by the blue line a) E. coli and $S$. aureus on stainless steel b) E. coli and $S$. aureus on $\mathrm{TiN} / 25.65$ at. $\% \mathrm{Ag}$. 

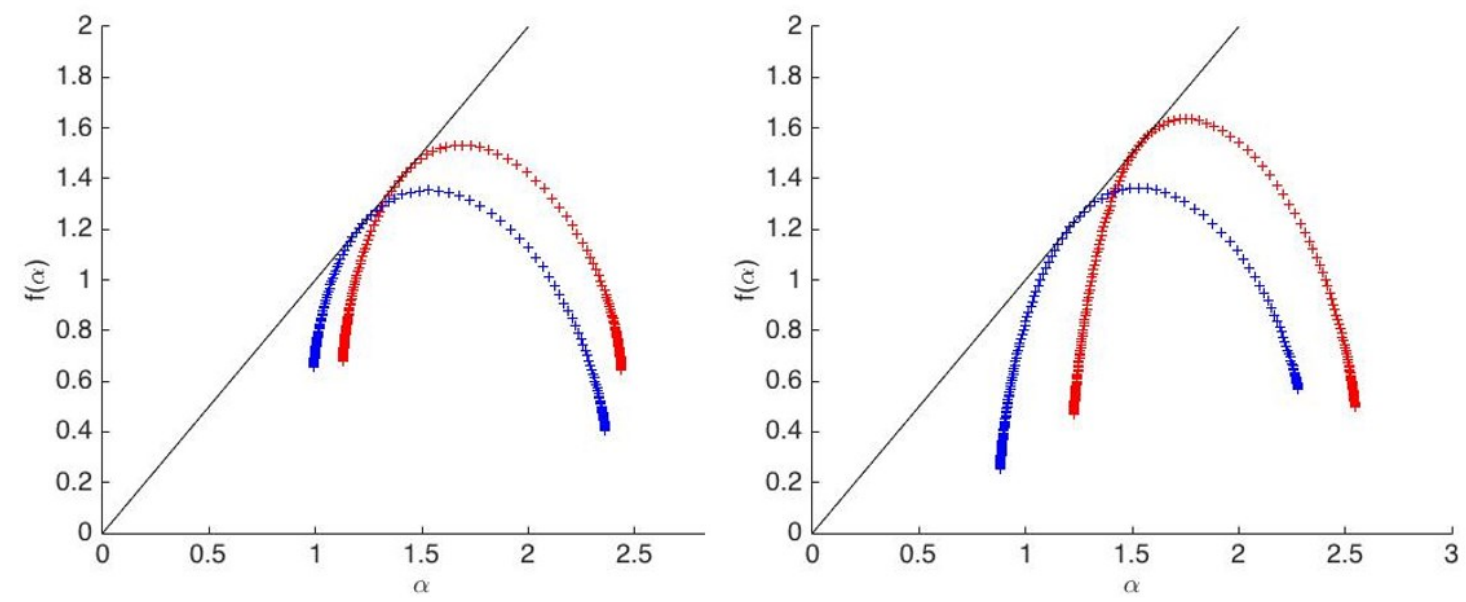

Fig. 5. Examples of $f$ alpha curves demonstrating how the data is extrapolated to describe the distribution and pattern of cells on the surfaces. E. coli is denoted by the red line and S. aureus is denoted by the blue line a) E. coli and $S$. aureus on stainless steel b) E. coli and $S$. aureus on $\mathrm{TiN} / 25.65$ at. $\% \mathrm{Ag}$. 

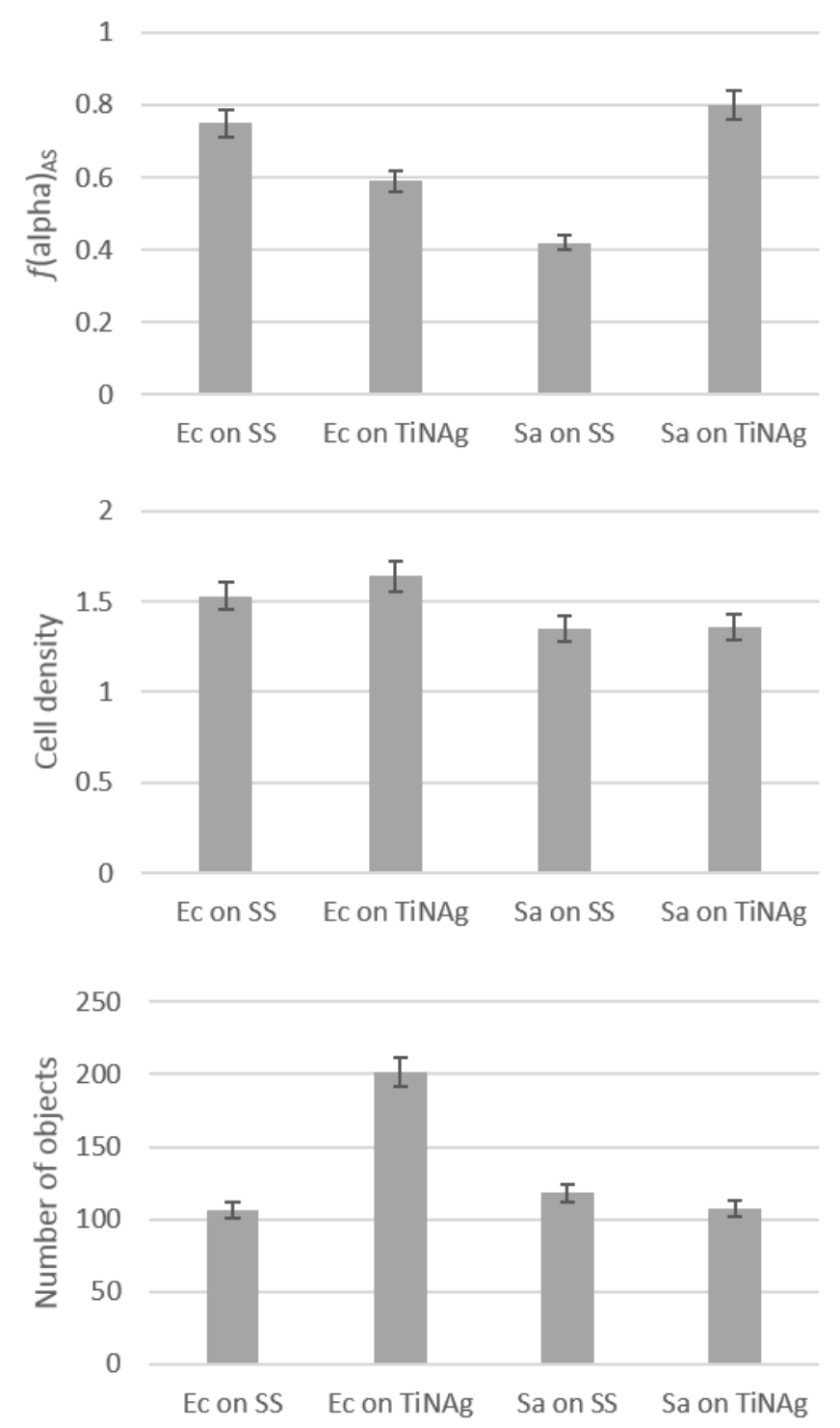

Fig. 6. Quantitative multifractal analysis data demonstrating the difference in a) homogeneity and heterogeneity spread b) density of the cells and c) number of cell clusters. 
Table 1. $S_{a}$, peak width and peak height values obtained by optical surface profiling upon the two substrates demonstrating that there was no significant difference in roughness values between the two surfaces.

\begin{tabular}{lll}
\hline Parameter & Stainless steel & TiN/25.65at. \% Ag \\
\hline$S_{a}(\mathrm{~nm})$ & $25.27 \pm 0.35$ & $29.06 \pm 2.13$ \\
Peak Width (nm) & $560-3210$ & $620-1900$ \\
Peak Height (nm) & $5.95-40.38$ & $10.43-48.19$ \\
\hline
\end{tabular}


Table 2. Measurements demonstrating the inhibitory effect of the stainless steel and TiN/25.65 at. \% Ag surfaces against S. aureus and E. coli.

\section{Zone of inhibition (cm) NVT (CFU)}

Stainless steel

E. coli

$\mathrm{TiN} / 25.65$ at. $\% \mathrm{Ag}$

E. coli

Stainless steel

S. aureus

TiN/25.65 at. $\% \mathrm{Ag}$

S. aureus
0

5.7

0

0

11

0

1

20 